\title{
An update on the spider genus Hexophthalma (Araneae: Sicariidae) in the Afrotropical region, with descriptions of new species
}

\author{
Leon N. LOTZ \\ Department of Arachnology, National Museum, P.O. Box 266, \\ Bloemfontein, 9300, South Africa. \\ Email: leonlotz57@gmail.com
}

urn:1sid:zoobank.org:author:D55D7B33-BAF0-48AC-B826-D3FF6787EEA7

\begin{abstract}
The described Afrotropical species of the genus Hexophthalma Karsch, 1879 (under the genus name Sicarius Walckenaer, 1847), of the spider family Sicariidae Keyserling, 1880, were recently reviewed. In the present paper the Afrotropical species of the genus Hexophthalma are revisited. After a thorough examination of all the available specimens from nine major collections, the species H. testacea (Purcell, 1908) is here synonymized with H. hahni (Karsch, 1878), three new species are described $H$. binfordae sp. nov., H. goanikontesensis sp. nov. (both from Namibia) and H. leroyi sp. nov. (from South Africa) - and the male of $H$. dolichocephala (Lawrence, 1928) is described for the first time. The distribution of the species is also revised and a new updated key to the species is compiled.
\end{abstract}

Keywords. Africa, six-eyed crab spiders, Haplogynae.

Lotz L.N. 2018. An update on the spider genus Hexophthalma (Araneae: Sicariidae) in the Afrotropical region, with descriptions of new species. European Journal of Taxonomy 424: 1-18. https://doi.org/10.5852/ejt.2018.424

\section{Introduction}

In a very recent paper on Sicariidae, Magalhaes et al. (2017) revalidated the genus name Hexophthalma Karsch, 1879 for six species occurring in Africa (World Spider Catalog 2017). The genus was firstly described as Hexomma (Karsch 1878) for the species H. hahni, but this name was preoccupied and replaced with Hexophthalma (Karsch 1879). Simon (1893) synonymized Hexophthalma with Sicarius Walckenaer, 1847. All subsequent authors published new Afrotropical species as Sicarius (Pocock 1900; Purcell 1908; Lawrence 1928). The Distribution of Hexophthalma is restricted to the southern part of the Afrotropical region. The majority of the species occur in the drier western half of southern Africa, extending eastward to the north. As very little collecting has been done in Angola, it is possible that the distribution may extend further north into this country.

In a recent paper on the Afrotropical species of Hexophthalma (Lotz 2012), a short review was given of the described species. This was based on the type specimens or specimens collected close to the type locality. Since then an intensive study of all available specimens from nine collections was undertaken. This led to the discovery of a lot of variation in the female genitalia and of unexpected prominent differences between the palps of the different species that can be used to differentiate between them. 
The dorsal setae on the leg femora were found to be too variable to be used as a diagnostic character for species, except in the case of H. spatulata (Pocock, 1900), where the setae are scoop shaped and situated on a slight mound (Fig. 23). In all other species the setae vary from thin to broad, with some having both thin and broad setae. In this publication the female genitalia and male palps are drawn from photos of actual specimens. In some cases the female genitalia of several specimens of the same species are depicted to show the intraspecific variation as well as the variation between the left and right side of the same specimen.

During the study three new species and the male of H. dolichocephala (Lawrence, 1928) were discovered and are described here. The variation in female genitalia and the differences found in the male palps also led to the realization that $H$. testacea (Purcell, 1908) and H. hahni (Karsch, 1878) is one and the same species and $H$. testacea is therefore here synonymized with $H$. hahni. The distribution of the different species is also updated here with maps showing the localities of the studied specimens only, because not all specimens mentioned in published records (Newlands 1986) could be found to corroborate their identification.

\section{Material and methods}

All the specimens that could be obtained on loan, from the collections mentioned below, were studied and are discussed here (responsible curator(s) mentioned in parentheses). Illustrations of the male palps and female genitalia were made from photos of the actual genitalia studied. Male palps were taken from the right side and studied and drawn from the prolateral side. Illustrations were made with CorelDRAW X4. Photos of the genitalia were taken with a Paxcam 5 digital camera, mounted on a Novel dissection microscope. Photos are not included, as they are not of publishable quality. All female genitalia and male palps were photographed and compared. Coordinates given in square brackets are not included on the specimen labels, but were looked up on physical maps and added.

\section{Abbreviations}

\section{Morphological terms}

$\begin{array}{ll}\mathrm{AME}-\mathrm{LE} & =\text { distance between anterior median and anterior lateral eyes } \\ \mathrm{CL} & =\text { carapace length } \\ \mathrm{CLL} & =\text { clypeus length } \\ \mathrm{CW} & =\text { carapace width } \\ \mathrm{TL} & =\text { total length }\end{array}$

\section{Institutions}

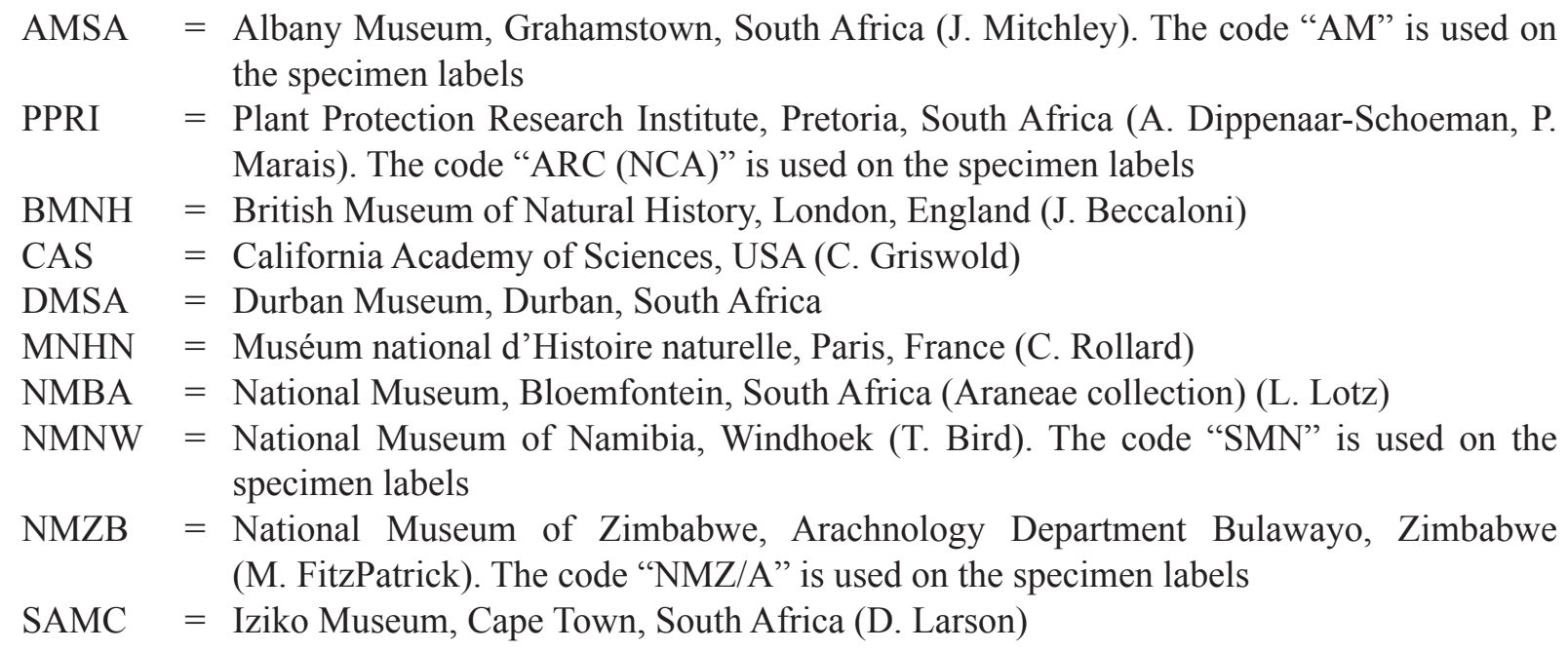




\title{
Results
}

\author{
Class Arachnida Cuvier, 1812
}

Order Araneae Clerck, 1757

Family Sicariidae Keyserling, 1880

Genus Hexophthalma Karsch, 1879

\section{Key to the species of Hexophthalma}

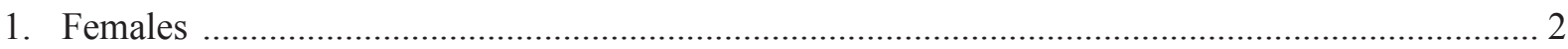

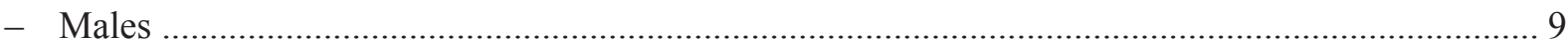

2. Enlarged dorsal setae on femora raised on a slight mound and scoop-shaped (Fig. 23); female spermathecae consist of numerous tubes, each ending in one or more spherical vesicles (Fig. 16) ..

H. spatulata (Pocock, 1900)

- Enlarged dorsal setae on femora not raised on a slight mound and not scoop-shaped; spermathecae consist of one or more finger-like tubes (Figs 1-15)

3. Inner spermathecae consist of one main finger-like tube on each side (Fig. 10)

..H. dolichocephala (Lawrence, 1928)

- Inner spermathecae consist of more than one finger-like tube on each side

4. Spermathecae without outer spermathecae near uterus externus and without reduced outer spermathecae (Fig. 9)

H. damarensis (Lawrence, 1928)

- Spermathecae with outer spermathecae near uterus externus or with reduced outer spermathecae ...

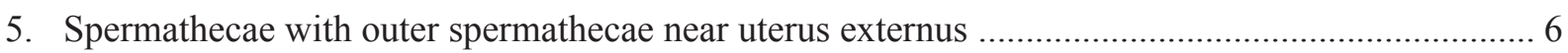

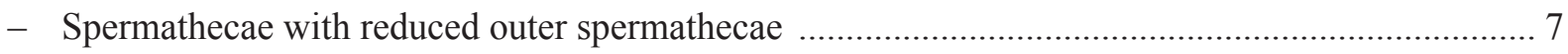

6. Inner spermathecae with long finger-like tubes (Figs 6-8) ...............H. albospinosa (Purcell, 1908)

- Inner spermathecae with shorter, more bulbous, finger-like tubes (Figs 11-15)

S. hahni (Karsch, 1878)

7. Inner spermathecae hammer-like, bent slightly laterally (Fig. 1)

H. binfordae sp. nov.

- Inner spermathecae not hammer-like, bent more or less medially

8. Inner spermathecae with two finger-like tubes, outer spermathecae relatively round (Fig. 5)

H. leroyi sp. nov.

- Inner spermathecae with three or more finger-like tubes, outer spermathecae long (Figs 2-4)

H. goanikontesensis sp. nov.

9. Embolus with a broad, blunt apex (Fig. 22)

H. spatulata (Pocock, 1900)

- Embolus with a narrow apex (Figs 17-21)

10. Embolus with a short, flattened apex (Fig. 20)

H. dolichocephala (Lawrence, 1928)

- Embolus with a thin, sharp apex

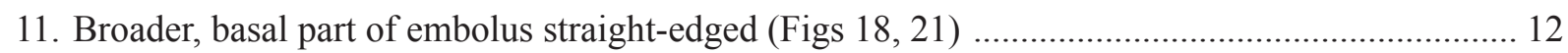

- Broader, basal part of embolus with a hump along one edge (Figs 17, 19) ................................... 13 
12. Thin apical part of embolus almost as long as thicker basal part (Fig. 17)

H. albospinosa (Purcell, 1908)

- Thin apical part of embolus much shorter than thicker basal part (Fig. 21)

H. hahni (Karsch, 1878)

13. Thin apical part of embolus without embolus keel at base (Fig. 19)

H. damarensis (Lawrence, 1928)

- Thin apical part of embolus with embolus keel at base (Fig. 18)

H. goanikontesensis sp. nov.

Hexophthalma binfordae sp. nov.

urn:1sid:zoobank.org:act:DD563DE2-A5BD-4AB0-8319-021E01B366DA

Figs 1, 24

\section{Diagnosis}

Female inner spermathecae consist of one lateral extending sac and a divided round sac medially, on each side, with a small sclerotized reduced outer spermatheca (Fig. 1) which is unlike that of any of the other species. Male unknown.

\section{Etymology}

The species name is a matronym for Greta Binford, collector of the type specimen.

\section{Material examined}

\section{Holotype}

NAMIBIA: + , Khorxas, Twyfelfontein, 18³0'53.64" S, 14²2'21.84” E, 19 Aug. 2005, G. Binford leg. (NMNW 45842).

\section{Description}

Female $(\mathrm{n}=1)$

$\mathrm{TL}=11.9 ; \mathrm{CL}=5.5 ; \mathrm{CW}=5.5 ; \mathrm{CLL}=0.7 . \mathrm{AME}-\mathrm{LE}=0.6$; eye diameter 0.2. Carapace reddish-brown, cephalic area slightly darker; abdomen and legs yellow-brown. Body and legs with lancet-like curved macrosetae, more strongly curved on body than on legs. Spermathecae consist of one lateral extending sac and a divided round sac medially, on each side, with a small sclerotized reduced outer spermatheca basally (Fig. 1).

Leg measurements:

\begin{tabular}{|lccccc|}
\hline & I & II & III & IV & Palp \\
\hline Femur & 7.0 & 7.2 & 7.2 & 7.0 & 1.6 \\
Patella & 2.6 & 2.6 & 2.5 & 2.3 & 1.0 \\
Tibia & 6.9 & 7.0 & 6.3 & 6.0 & 1.0 \\
Metatarsus & 4.8 & 4.7 & 4.6 & 4.6 & - \\
Tarsus & 2.9 & 3.0 & 2.8 & 3.0 & 2.1 \\
Total & 24.2 & 24.5 & 23.5 & 22.9 & 5.7 \\
\hline
\end{tabular}

\section{Natural history}

Hexophthalma binfordae sp. nov. was collected from a savanna area.

\section{Distribution}

Hexophthalma binfordae sp. nov. is only known from the type locality in Namibia (Fig. 24). 
LOTZ L.N., Spider genus Hexophthalma in the Afrotropical region

Hexophthalma goanikontesensis sp. nov. urn:1sid:zoobank.org:act:EA012B80-652F-43DF-B071-33618A497072

Figs 2-4, 18, 24

\section{Diagnosis}

Female inner spermathecae consist of sac-like fingers on each side, with a sclerotized reduced outer spermatheca; the number of sac-like spermathecae varies from three to seven between specimens and from side to side (Figs 2-4); may at first glance resemble H. leroyi sp. nov., but with more than two spermathecae sacs, the other sacs hidden behind two top sacs. Male embolus with a thin apical part with small keel at the base and a thicker basal part with a slight hump on one side, similar to that of $H$. damarensis, but differing by the presence of the small keel and the side hump being apical of median, on the thicker part of the embolus (Fig. 18).

\section{Etymology}

The specific name is an adjective derived from the type locality.

\section{Material examined}

\section{Holotype}

NAMIBIA: ${ }^{\top}$, Swakpomund, Namib-Naukluft Park, Goanikontes, $22.68565^{\circ}$ S, $14.84964^{\circ}$ E, 7 Feb.28 Mar. 2009, J. Irish \& R. Scholtz leg. (NMNW 47282).

\section{Paratypes}

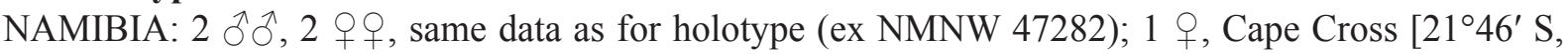
135'ㄹ' E], 8 Jul. 1963, A. Els leg. (NMNW 35066); 2 우옹 Damaraland, Klein Graias, 2014Cc, 5 Aug. 1985, E. Griffin leg. (NMNW 41688); 1 đ’, 1 imm., Kaokoland, $17^{\circ} 37^{\prime}$ S, $12^{\circ} 12^{\prime}$ E, 14 Oct. 1988 ,

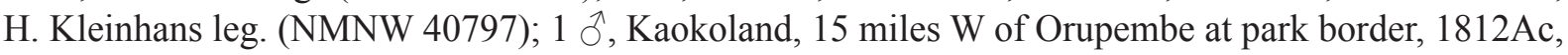
R.E.G. leg. (NMNW 35964); 2 + 9,3 imm., Koichab River, Sceleton coast, 20 miles inland [ca 20 $21^{\prime}$ S, $13^{\circ} 33^{\prime}$ E], 8 Nov. 1968, R.F. Lawrence leg. (AMSA); 1 , , Rössing Mine, Arandis water, 2214Bd, 7 Jun. 1984, E. Griffin leg. (NMNW 38930); 1 Ĵ, Rössing Mine, Crusher Dust area, 2215Ca, 19 Mar.-9 Apr.

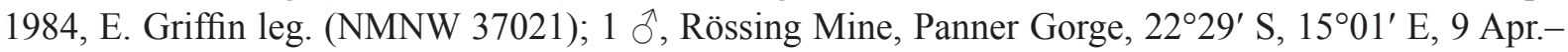
8 May 1984, E. Griffin leg. (NMNW 37086); 1 ㅇ, same data as for preceding but 11 Mar.-9 Apr. 1985,

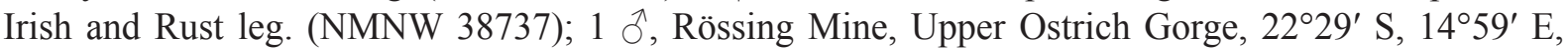
9 Apr.-8 May 1984, E. Griffin leg. (NMNW 37057); 1 ô, same data as for preceding but 5 Apr.-6 May 1985, Irish and Rust leg. (NMNW 38794); 1 ऽ, Swakopmund, $22.67^{\circ} \mathrm{S}, 14.52^{\circ} \mathrm{E}, 15$ Mar. 2010,

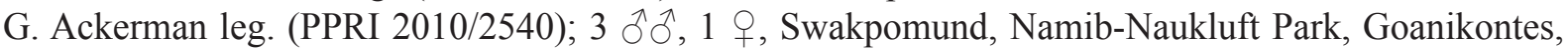
22043'11.9" S, 1449'23.2" E, 7-28 Feb. 2009, J. Irish \& R. Scholtz leg. (NMNW 47221, 47233); 1 , same data as for preceding but 5 Feb. 1929, R.D. Bradfield leg. (AMSA 6003); 1 , Ugab River

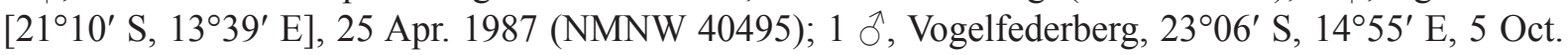
1984, E. Griffin leg. (NMNW 42837).

\section{Note}

Hexophthalma goanikontesensis sp. nov. appears to be the same species as $H$. sp. 2 in Magalhaes et al. (2017), judging from the figures in their publication (figs 20C-D, 21C-D).

\section{Description}

Female $(\mathrm{n}=7)$

$\mathrm{TL}=10.26$ (8.8-12.4); $\mathrm{CL}=4.80$ (3.7-6.0); $\mathrm{CW}=4.76$ (3.8-5.6); $\mathrm{CLL}=0.59$ (0.5-0.8). AME-LE=0.6; eye diameter $=0.2$. Carapace reddish-brown, cephalic area slightly darker; abdomen and legs yellowbrown. Body and legs with lancet-like curved macrosetae, more strongly curved on body than on legs. Inner spermathecae consist of sac-like fingers on each side, with a sclerotized reduced outer spermatheca; 
the number of sac-like inner spermathecae varies from three to seven sacs among specimens and from side to side (Figs 2-4).

Leg measurements:

\begin{tabular}{|lccccc|}
\hline & I & II & III & IV & Palp \\
\hline Femur & 6.0 & 6.3 & 6.0 & 6.2 & 1.8 \\
Patella & 2.3 & 2.4 & 2.3 & 2.3 & 1.0 \\
Tibia & 5.5 & 5.6 & 5.1 & 5.1 & 1.0 \\
Metatarsus & 3.9 & 4.0 & 3.5 & 3.8 & - \\
Tarsus & 2.8 & 2.6 & 2.5 & 2.8 & 1.9 \\
Total & 20.5 & 20.9 & 19.4 & 20.2 & 5.7 \\
\hline
\end{tabular}

Male $(\mathrm{n}=10)$

$\mathrm{TL}=9.01$ (8.0-9.8); $\mathrm{CL}=4.65$ (4.1-5.2); $\mathrm{CW}=4.62$ (4.2-5.1); $\mathrm{CLL}=0.61$ (0.60-0.65). AME-LE=0.55; eye diameter $=0.2$. Colour and spination as in female. Embolus with a thin apical part with small keel at the base and a thicker basal part with a slight hump on one side, apical of median on the thicker part of the embolus (Fig. 17).

Leg measurements:

\begin{tabular}{|lccccc|}
\hline & I & II & III & IV & Palp \\
\hline Femur & 6.3 & 7.0 & 6.4 & 6.4 & 1.6 \\
Patella & 2.2 & 2.2 & 2.2 & 2.0 & 1.0 \\
Tibia & 5.8 & 5.9 & 5.5 & 5.4 & 1.2 \\
Metatarsus & 4.1 & 4.3 & 4.0 & 3.8 & - \\
Tarsus & 2.8 & 2.8 & 2.6 & 2.6 & 0.6 \\
Total & 21.2 & 22.2 & 20.7 & 20.2 & 4.4 \\
\hline
\end{tabular}

\section{Natural history}

Hexophthalma goanikontesensis sp. nov. have been collected in desert areas, by pit traps and by hand from under rocks.

\section{Distribution}

Hexophthalma goanikontesensis sp. nov. is known from the northern coastal areas in Namibia (Fig. 24).

Hexophthalma leroyi sp. nov. urn:1sid:zoobank.org:act:3EAAF34D-D7DB-4C5F-84BE-231233E064B4

\section{Diagnosis}

Figs 5, 24

Female inner spermathecae consist of two sac-like fingers on each side, with a round sclerotized reduced outer spermatheca (Fig. 5), to a certain extent similar to H. goanikontesensis sp. nov., but with only two inner spermathecae sacs. Male unknown.

\section{Etymology}

The specific name is a patronym for John Leroy, collector of the type specimen. 


\section{Material examined}

\section{Holotype}

SOUTH AFRICA: + , Northern Cape Province, Augrabies National Park, 28.66 S, 20.42 ${ }^{\circ}$ E, 24 Feb. 1983, J. Leroy leg., LR270 (PPRI 89/786).

\section{Description}

Female $(\mathrm{n}=1)$

$\mathrm{TL}=12.9 ; \mathrm{CL}=5.9 ; \mathrm{CW}=6.0 ; \mathrm{CLL}=0.9 . \mathrm{AME}-\mathrm{LE}=0.65 ;$ eye diameter $=0.2$. Carapace reddish-brown, cephalic area slightly darker; abdomen and legs yellow-brown. Body and legs with lancet-like curved macrosetae; macrosetae more curved on body than on legs. Inner spermathecae consist of two sac-like fingers on each side, with a round, sclerotized, reduced outer spermatheca (Fig. 5).

Leg measurements:

\begin{tabular}{|lccccc|}
\hline & I & II & III & IV & Palp \\
\hline Femur & 7.2 & 7.4 & 6.8 & 7.0 & 1.6 \\
Patella & 2.6 & 2.8 & 2.7 & 2.7 & 1.0 \\
Tibia & 7.1 & 7.1 & 6.8 & 6.2 & 1.1 \\
Metatarsus & 4.9 & 4.8 & 4.3 & 4.6 & - \\
Tarsus & 2.8 & 2.8 & 2.6 & 3.2 & 2.0 \\
Total & 24.6 & 24.9 & 23.2 & 23.7 & 5.7 \\
\hline
\end{tabular}

\section{Natural history}

Hexophthalma leroyi sp. nov. was collected in a grassy area in the Nama Karoo biome.

\section{Distribution}

Hexophthalma leroyi sp. nov. is known only from the type locality in South Africa (Fig. 24).

Hexophthalma albospinosa (Purcell, 1908)

Figs $6-8,17,25$

Sicarius albospinosus Purcell, 1908: 224.

Sicarius albospinosus - Lawrence 1938: 215. — Newlands 1986: 45. — Lotz 2012: 3.

Hexophthalma albospinosa - Magalhaes et al. 2017: 851, figs 20A-B, 21A-B, 62A-C.

\section{Revised diagnosis}

Female inner spermathecae consist of long finger-like sacs that branch close to the copulatory tube end, similar to in H. hahni except that the finger-like tubes are longer and thinner (Figs 6-8). Male embolus consisting of a thin pointed apical part and straight-sided thicker basal section (Fig. 17), similar to in H. hahni except for the longer thin apical part in relation to the thicker basal part.

\section{Material examined}

\section{Lectotype}

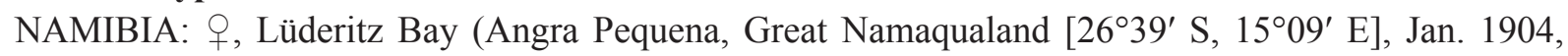
L. Schultze leg. (SAMC X150521). 


\section{Paralectotypes}

NAMIBIA: 2 juv., same data as for lectotype (SAMC X150521).

\section{Other material}

NAMIBIA: 1 ðे, Agate Beach, 2615Ca, 9 Oct. 1984, M. Griffin leg. (NMNW 42838); 1 q, same data as

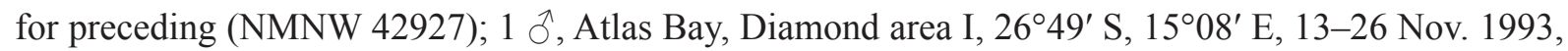

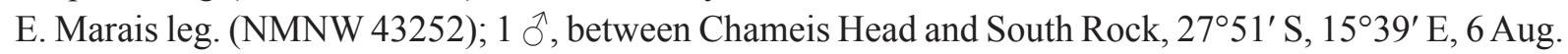
1997, E. Griffin leg. (NMNW 43810); 1 ㅇ, 1 imm., Griffiths Bay, Diamond area 1, 26³9' S, $1^{\circ} 07^{\prime}$ E, 16 Jun. 1993, M. Griffin leg. (NMNW 42894); 1 ð̃, Jammerbucht, $27^{\circ} 10^{\prime}$ S, $15^{\circ} 16^{\prime}$ E, 20 Jun. 1993, M. Griffin leg. (NMNW 42912); 1 §̊, 3 우, 4 imm., Klinghardts Mountain foothills, Diamond area 1,
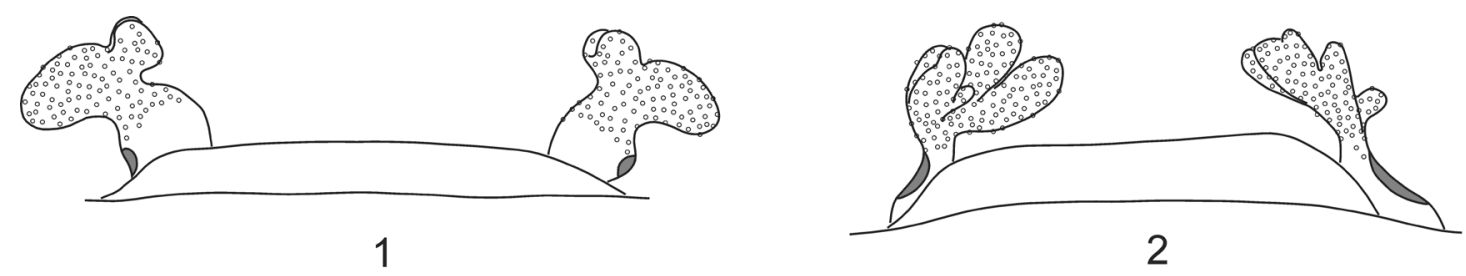

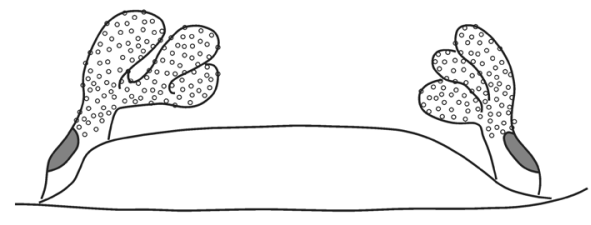

3

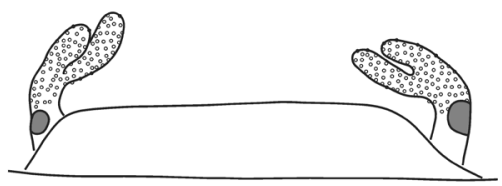

5

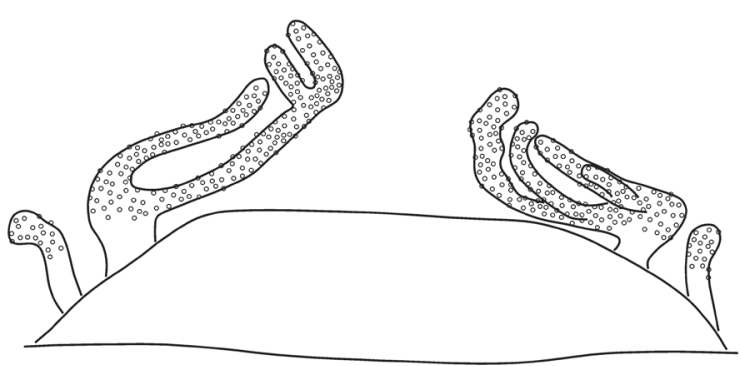

7

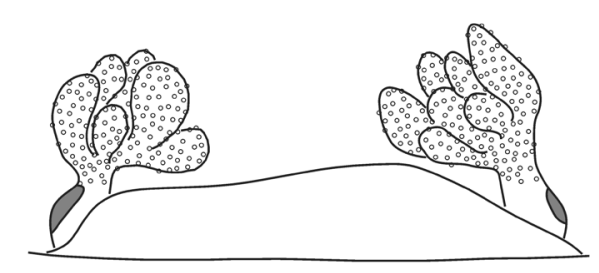

4

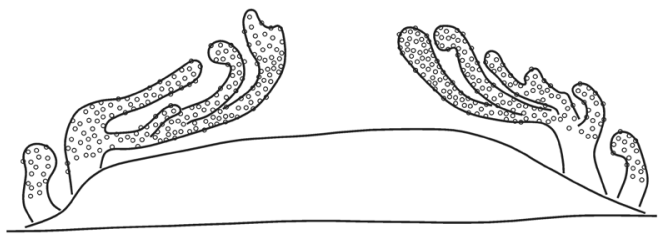

6

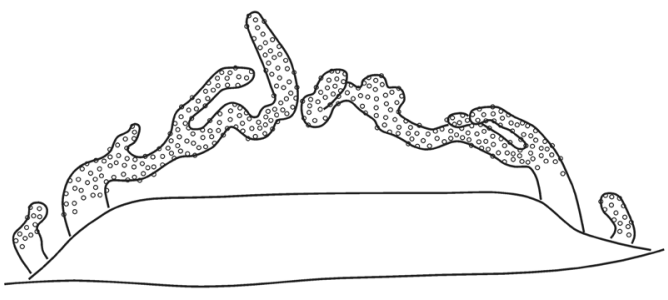

8

Figs 1-8. Female internal genitalia. 1. Hexophthalma binfordae sp. nov. 2-4. H. goanikontesensis sp. nov. 5. H. leroyi sp. nov. 6-8. H. albospinosa (Purcell, 1908). Scale bar $=1 \mathrm{~mm}$. 


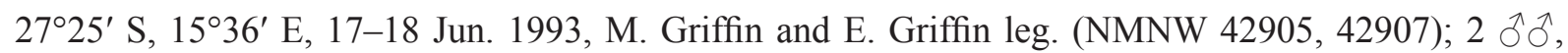

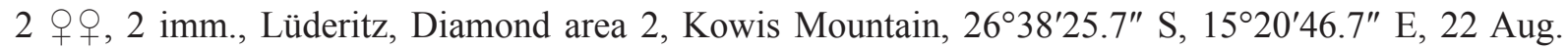
2006, EduVentures 9 leg. (NMNW 47257, 47258); 1 ô, Lüderitz, Lüderitz Peninsula, 26³9'58.5" S, 1507'19.9" E, 14 Dec. 2008-3 Feb. 2009, I. Wiesel, T.L. Bird and C. Bird leg. (NMNW 47339); 1 ð,

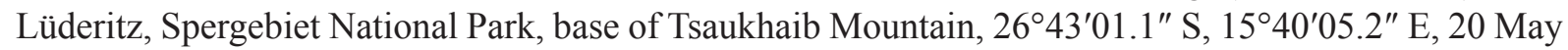
2009, EduVentures leg. (NMNW 47367); 1 \%, NamDeb southern mine, old articulated dumptruck, 30 Jan.-12 Feb. 1997, V. Ward and J. Daneel leg. (NMNW 43565); 2 우, Oranjemund, 28.55 $\mathrm{S}$, $16.43^{\circ}$ E, Jan. 1991, N. Larson leg. (PPRI 91/1530); 1 \%, Oranjemund site 3, NamDeb mine dumps, 2816Cb, 23-26 Jan. 1998, V. Ward leg. (NMNW 43844); 1 §, Oranjemund mining area, control site 5, near Orange River, 20 Sept.-2 Oct. 1996, E. Griffin leg. (NMNW 43554); 1 \%, Orange River Mouth, coastal dunes, 2826Cb, 10 Nov. 1986, E. Griffin leg. (NMNW 42957).

\section{Natural history}

Hexophthalma albospinosa have been collected in the Succulent Karoo biome, by pitfall traps and by hand from under rocks and old roofing tin.

\section{Distribution}

Hexophthalma albospinosa is distributed along the southern coastal areas of Namibia (Fig. 25).

Hexophthalma damarensis (Lawrence, 1928)

Figs 9, 19, 25

Sicarius damarensis Lawrence, 1928: 221.

Sicarius damarensis - Newlands 1986: 47. — Lotz 2012: 4.

Hexophthalma damarensis - Magalhaes et al. 2017: 852.

\section{Diagnosis}

Female inner spermathecae unique, with short, bulbous, finger-like sacs that branch off the copulatory tube part, without reduced outer spermathecae (Fig. 9). Male embolus ending in a thin, pointed apex; thicker basal part of embolus with a hump on one side, slightly basal from median (Fig. 19); this is similar to in H. goanikontesensis sp. nov. except that the thinner apical part lacks a basal embolus keel and the hump on the side of the thicker part is more basal.

\section{Material examined}

Holotype

NAMIBIA: , , Outjo $\left[20^{\circ} 07^{\prime} \mathrm{S}, 16^{\circ} 09^{\prime} \mathrm{E}\right](\mathrm{SAMC} 7057)$.

\section{Other material}

NAMIBIA: $1 \partial^{\top}$, Helio, 1903' S, 16²9’ E, 14 Feb.-23 Mar. 1987, E. Griffin leg. (NMNW 40160); 1 q, $1 \mathrm{imm}$., same data as for preceding but 8 Aug. 1987-27 Mar. 1988 (NMNW 40986); 1 đ̊, same data as for preceding but 27 Mar.-4 May 1988 (NMNW 41135).

\section{Natural history}

Hexophthalma damarensis have been collected in Savanna biomes using pitfall traps.

\section{Distribution}

Hexophthalma damarensis is distributed in the northern central part of Namibia (Fig. 25). 
Hexophthalma dolichocephala (Lawrence, 1928)

Figs 10, 20, 25

Sicarius dolichocephalus Lawrence, 1928: 222.

Sicarius dolichocephalus - Newlands 1986: 49. — Lotz 2012: 8.

Hexophthalma dolichocephala - Magalhaes et al. 2017: 850.

\section{Diagnosis}

Female inner spermathecae consist of one finger-like sac on each side, with a very short outer spermatheca (Fig. 10). Male embolus apical part thin, blunt and slightly twisted and flattened, unlike the other species where the apical part is thin and pointed (Fig. 20).

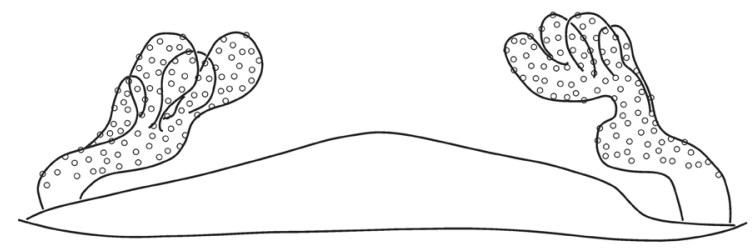

9

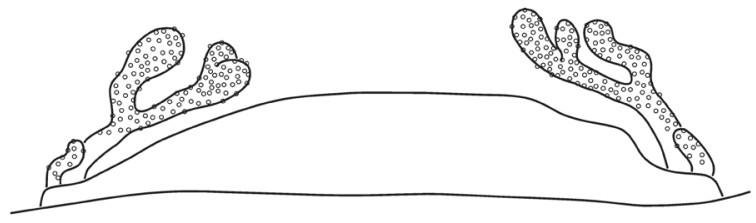

11

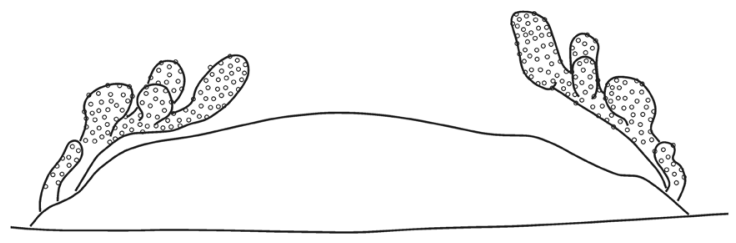

13

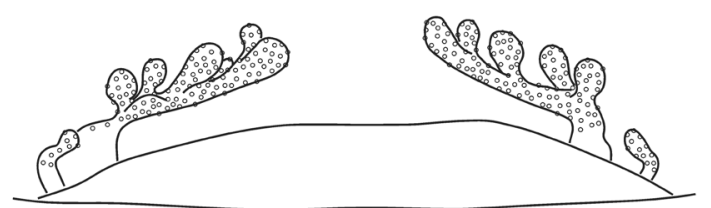

15

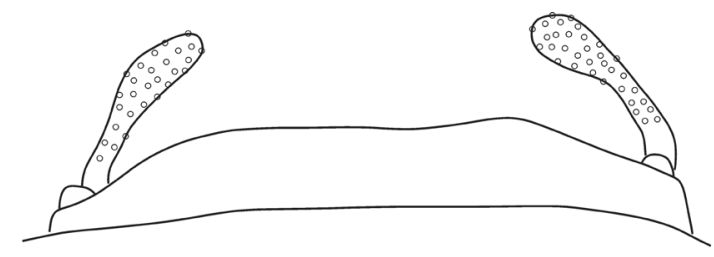

10

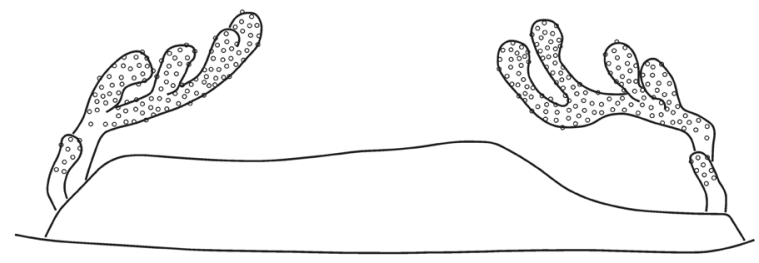

12

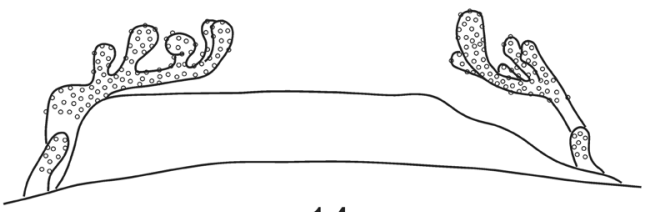

14

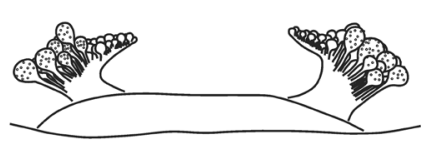

16

Figs 9-16. Female internal genitalia. 9. Hexophthalma damarensis (Lawrence, 1928). 10. H. dolichocephala (Lawrence, 1928). 11-15. H. hahni (Karsch, 1878). 16. H. spatulata (Pocock, 1900). Scale bar $=1 \mathrm{~mm}$. 


\section{Material examined}

\section{Lectotype}

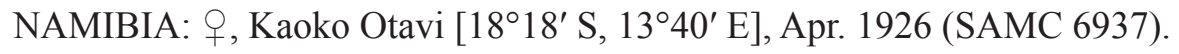

\section{Other material}

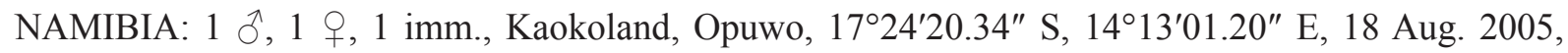

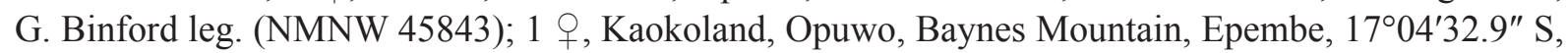

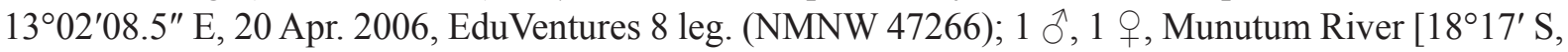
$12^{\circ} 04^{\prime} \mathrm{E}$ ], 19 Oct. 1988 , H. Kleinhans leg. (NMNW 40863); 1 ' , Okahama water point, $17^{\circ} 10^{\prime} 34.1^{\prime \prime} \mathrm{S}$, 12 59'54.7" E, 25 Apr. 2006, EduVentures 8 leg. (NMNW 47271); $1 \partial^{\wedge}$, Möwe Bay, $19^{\circ} 20^{\prime} \mathrm{S}, 12^{\circ} 40^{\prime} \mathrm{E}$,

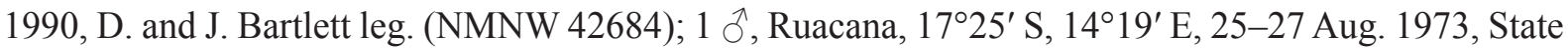
Museum leg. (NMNW 35801).

\section{Description}

Male ( $\mathrm{n}=5)$

$\mathrm{TL}=19.5$ (11.6-28.7); $\mathrm{CL}=4.98$ (4.7-5.6); $\mathrm{CW}=4.94$ (4.7-5.6); $\mathrm{CLL}=0.59$ (0.50-0.65). AME$\mathrm{LE}=0.5$; eye diameter $=0.2$. Carapace reddish-brown, cephalic area slightly darker; abdomen and legs yellow-brown. Body and legs with lancet-like curved macrosetae, more strongly curved on body than on legs. Embolus thick over most of the length, tapered apically, point blunt and slightly twisted at base of thinner part (Fig. 20).

Leg measurements:

\begin{tabular}{|lccccc|}
\hline & I & II & III & IV & Palp \\
\hline Femur & 7.4 & 8.6 & 7.4 & 7.7 & 1.5 \\
Patella & 2.3 & 2.4 & 2.2 & 2.2 & 0.9 \\
Tibia & 8.4 & 8.8 & 7.6 & 7.3 & 1.2 \\
Metatarsus & 6.2 & 7.5 & 6.5 & 6.5 & - \\
Tarsus & 2.9 & 3.3 & 2.9 & 3.3 & 0.7 \\
Total & 27.2 & 30.6 & 26.6 & 27.0 & 4.3 \\
\hline
\end{tabular}

\section{Natural history}

Hexophthalma dolichocephala have been collected in Desert, Nama Karoo and Savanna biomes, by pitfall traps and by hand from under rocks.

\section{Distribution}

Hexophthalma dolichocephala is distributed in the northwestern corner of Namibia (Fig. 25).

\section{Hexophthalma hahni (Karsch, 1878)}

Figs 11-15, 21, 26

Hexomma hahnii Karsch, 1878: 325.

Sicarius testaceus Purcell, 1908: 224. Syn. nov.

Hexophthalma hahni - Karsch, 1879: 109. - Magalhaes et al. 2017: 850, figs 13B, 14B-C, 16G, 18G, 19C, 23A-C, 24A, 61A-D.

Sicarius hahni-Simon, 1893: 269. — Purcell 1908: 225. — Newlands 1986: 51. — Lotz 2012: 8. 
Sicarius testaceus - Newlands 1986: 57. — Lotz 2012: 12.

Hexophthalma testacea - Magalhaes et al. 2017: 852.

Note: Sicarius oweni Newlands, 1986: 53 is an unpublished synonym.

\section{Diagnosis}

Female inner spermathecae consist of finger-like sacs that branch close to the copulatory tube end, similar to in H. albospinosa except that the finger-like tubes are shorter and more bulbous (Figs 11-15). Male embolus consisting of a thin pointed apical part and a straight-sided thicker basal section (Fig. 21), similar to in H. albospinosa except that the thin apical part is shorter in relation to the thicker basal part.
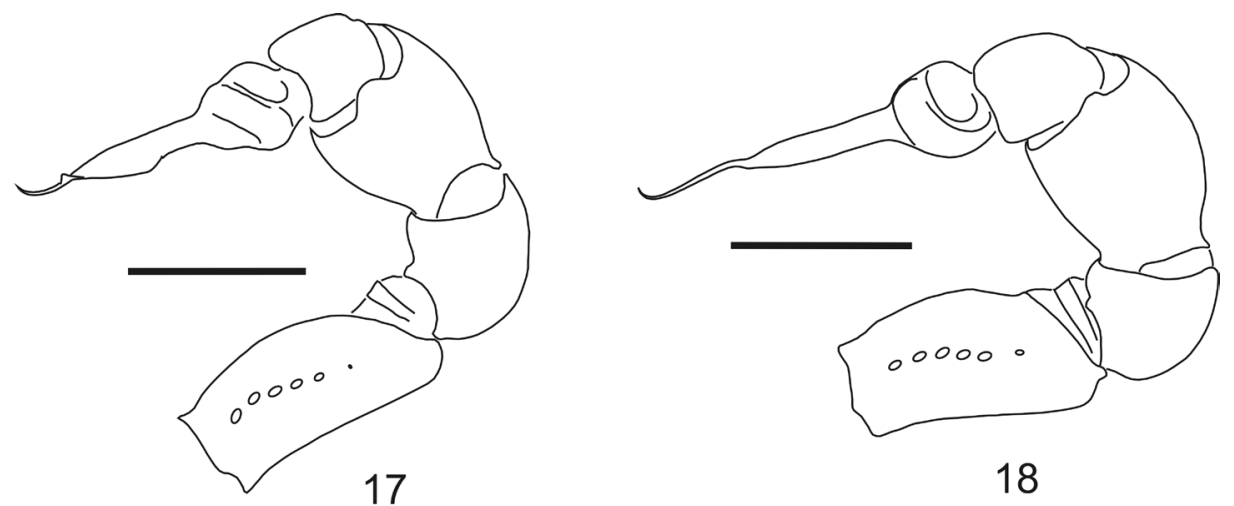

18

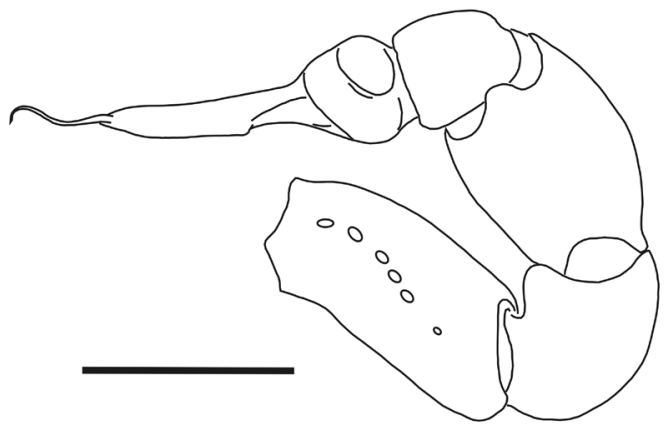

19

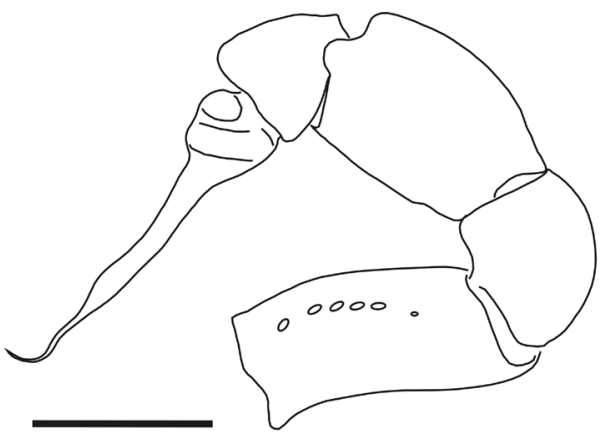

21

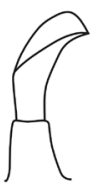

23

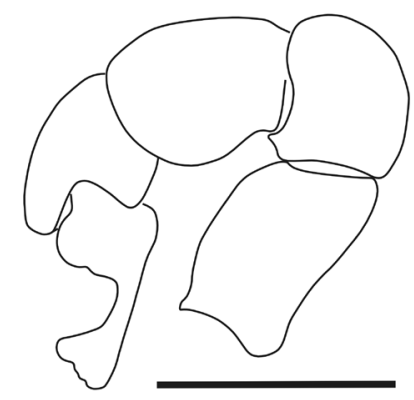

22

Figs 17-23. 17-22. Male palps (right side prolateral). 17. Hexophthalma albospinosa (Purcell, 1908). 18. H. goanikontesensis sp. nov. 19. H. damarensis (Lawrence, 1928). 20. H. dolichocephala (Lawrence, 1928). 21. H. hahni (Karsch, 1878). 22. H. spatulata (Pocock, 1900). 23. H. spatulata, enlarged femoral seta (not to scale). Scale bars $=1 \mathrm{~mm}$. 


\section{Material examined}

COUNTRY UNKNOWN: 2 ふึ, 2 우, 4 imm. (SAMC B7732, B8870).

NAMIBIA: $1 \overbrace{}^{\lambda}$ [no locality or date], G. Brown leg. (SAMC B5704); $1 \partial^{\lambda}$, Ganab, $23^{\circ} 04^{\prime} \mathrm{S}, 15^{\circ} 10^{\prime} \mathrm{E}, 13-$ 16 Sep. 1971, P.G. Olivier leg. (NMNW 35311); 1 q, same data as for preceding (NMNW 35313); 1 q, Grootfontein, Nosib Cave, 1917Bd, 8 Feb. 1995, SEGL leg. (NMNW 43454); 1 o, 1 + , Keetmanshoop, Khabus 146, 2618Ac, 7 Dec. 1987-17 Jan. 1988, Mar. 1992, N. and G. Olivier leg. (NMNW 40752); 2

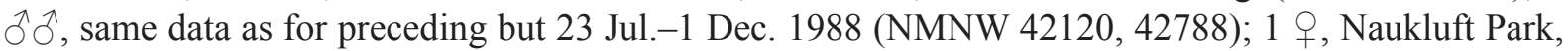
Gifgat 2, 2215Da, 11 Jul. 1995, E. Marais leg. (NMNW 43673); 1 , 1 imm., Naukluft Park, Gobabeb, $23^{\circ} 33^{\prime} 28.2^{\prime \prime} \mathrm{S}, 15^{\circ} 02^{\prime} 14.32^{\prime \prime} \mathrm{E}, 25$ Oct. 1968, R.F. Lawrence leg. (AMSA); 1 ð, 2 우, same data as for preceding but 21 Aug. 2005, G. Binford leg. (NMNW 45835); 1 § , 2 웅, Naukluft Park, Kuiseb River, near Gobabeb [233' S, $1^{\circ} 03^{\prime}$ E], 18 Feb.-20 Mar. 1983, Kuiseb survey leg. (PPRI 83/341); $1 \widehat{\jmath}$, same data as for preceding but 27 Aug. 1985, C.S. Crawford leg. (NMNW 41116); 1 ô, 1 q, Otjiwarongo, Waterberg Plateau Park, 20³0'17.94" S, 17014'32.16" E, 14 Aug. 2005, G. Binford leg. (NMNW 45838); 1 đo, Sossusvlei, $24.7^{\circ} \mathrm{S}, 15.42^{\circ}$ E, Dec. 1988, B. Grobbelaar leg. (PPRI 92/54);

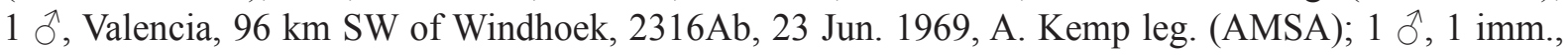
Windhoek, Daan Viljoen Game Reserve, 22 ${ }^{\circ} 32^{\prime} 16.14^{\prime \prime}$ S, 16 ${ }^{\circ} 54^{\prime} 44.16^{\prime \prime}$ E, 12 Aug. 2005, G. Binford leg. (NMNW 45845); 1 ô, Windhoek, Verloren Cave, $23^{\circ} 09^{\prime} \mathrm{S}, 16^{\circ} 20^{\prime} \mathrm{E}, 28$ Jun. 1993, J. Irish leg. (NMBA 7361); 1 ð’, 1 imm., Messum Crater, $21^{\circ} 20.430^{\prime}$ S, $14^{\circ} 13.218^{\prime}$ E, 4-10 Apr. 2000, E. Griffin leg.

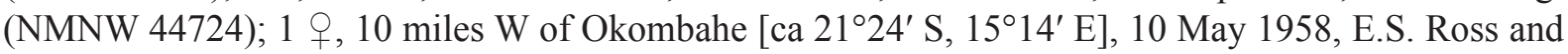
R.E. Leech leg. (CAS).

SOUTH AFRICA - Limpopo Province: 1 $\widehat{\text {, }}$, Kruger National Park, Pafuri area, $22.46^{\circ} \mathrm{S}, 31.3^{\circ} \mathrm{E}$, 14 Oct. 1995, A. Leroy leg. (PPRI 98/405); 1 + , Limpopo Valley National Park, $22.22^{\circ} \mathrm{S}, 29.13^{\circ} \mathrm{E}, 29$ Apr. 1997, J. Leroy leg. (PPRI 97/816); $4 \overbrace{}^{\lambda}{ }^{\lambda}$, Makgabeng area, $\mathrm{W}$ of Senwabawana, $23.24^{\circ} \mathrm{S}, 28.85^{\circ} \mathrm{E}$, 22 Dec. 2006, M. Burger and R. Hawkins leg. (PPRI 2008/3788, 2008/3807); 1 o, Rochdale, near Waterpoort, $22.54^{\circ} \mathrm{S}, 29.41^{\circ} \mathrm{E}, 4$ Apr. 1993, A. Leroy leg. (PPRI 2007/1626); 1 ${ }^{\lambda}$, Vhembi Biosphere, Gondeni, $22.914^{\circ} \mathrm{S}, 30.050^{\circ}$ E, 4 Dec. 2012, C. Schoeman leg. (PPRI 2015/632). - Mpumalanga: 1 , 2 imm., Bango Poort, Cave Poort, 24.58 ${ }^{\circ}$ S, 31.1 ${ }^{\circ}$ E, Mar. 1984, L. Braak leg. (PPRI 85/174). - Northern Cape Province: $1{ }^{\lambda}$, Blackridge, E of Langberge, E-NE of Groblershoop, $28^{\circ} 49^{\prime} \mathrm{S}$, $22^{\circ} 32^{\prime}$ E, 22 Dec. 2005, M. Burger leg. (PPRI 2009/3835); 1 đ̊, Bo-Seekoebaard, SE of Groblershoop

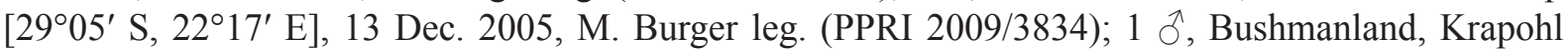
Island [28 50'30" S, 1843'30" E], 1909 (SAMC 14708); 2 우, 4 imm., Calvinia, Klein Arendskraal,

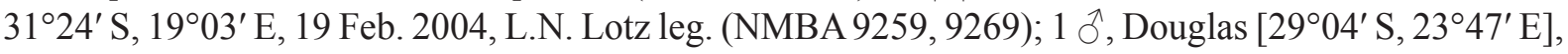

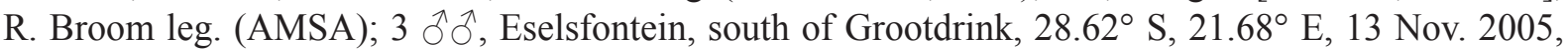
M. Burger, P. Braad and A. Hill leg. (PPRI 2009/3830-32); 1 §, same data as for precedings but 22 Nov. 2005, M. Burger leg. (PPRI 2009/3833); 1 ^, 1 imm., Little Namaqualand, Kamies [Kamieskroon?] [ca $30^{\circ} 13^{\prime} \mathrm{S}, 17^{\circ} 56^{\prime} \mathrm{E}$ ] (SAMC B7442); $10^{\top}, 1$ imm., Namaqualand, Kap Kap 382, 30 $10^{\circ} \mathrm{S}, 18^{\circ} 22^{\prime} \mathrm{E}$, 20 Oct. 1990, L.N. Lotz leg. (NMBA 5122); 1 đ̃, 1 क , Nieuwoudtville, Oorlogskloof Nature Reserve, 31.45 S, $19.1^{\circ}$ E, 27 Apr. 2000, A. Leroy leg. (PPRI 2010/187); 1 ㅇ, 2 imm., NW Gordonia, C.A. Anderson leg. (AMSA 1967); 1 đ̊, Port Nolloth, 2916Bd, 13 Mar. 1973, M.-L. Penrith leg. (NMNW

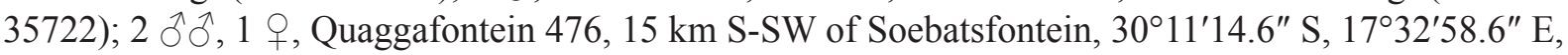
29 Sept. 2002, BIOTA leg. (PPRI 2009/5561, 2009/5562). - North West Province: 1 , Groot Marico, 25.6 ${ }^{\circ} \mathrm{S}, 26.43^{\circ} \mathrm{E}, 21$ Jan. 1991, M. Filmer leg. (PPRI 91/1323). - Western Cape Province: $1 q, 10 \mathrm{~km}$ $\mathrm{N}$ of Bitterfontein [ca 30 59' S, $18^{\circ} 14^{\prime}$ E], 15 Sept. 1983, J.V. leg. (SAMC C1981); 1 क , Clanwilliam District (SAMC 3548); 1 o, Clanwilliam, Sanddrif (Dwarsrivier 330), 32.16 S, $18.89^{\circ}$ E, Feb. 1993, G. Müller leg. (PPRI 93/261); 1 \&, Donkins Bay, 3118Cd, 14 Mar. 1973, M.J. and M.-L. Penrith leg.

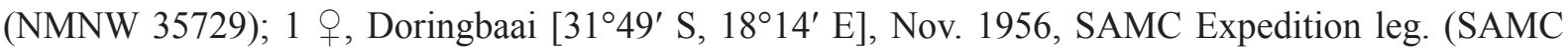
C1235); 3 우오, Swartberg Nature Reserve, Gamkaskloof, 33.36 S, $21.69^{\circ}$ E, Dec. 1995, M. de Jager leg. (PPRI 96/399); 1 †, 12 imm., same data as for preceding but 1 Jul. 2000, Z. van der Walt leg. 
(PPRI 2002/44); 1 9, Hans Strydom Dam Nature Reserve, Mogol, 33.95 ${ }^{\circ}$ S, $22.46^{\circ}$ E, 19 Jan. 1983 , M. Mansell leg. (PPRI 83/198); 1 đo, The Island, S of Herbertsdale, $34.08^{\circ} \mathrm{S}, 21.77^{\circ} \mathrm{E}, 3 \mathrm{Jan} .2006$, M. Burger leg. (PPRI 2009/3828); 1 ${ }^{\top}$, same data as for preceding but 30 Jan. 2006 (PPRI 2009/3829); 1 †, $19 \mathrm{~km} \mathrm{SE}$ of Klawer [ca 31 $54^{\prime} \mathrm{S}, 18^{\circ} 39^{\prime} \mathrm{E}$ ], 1 May 1958 (CAS); 1 q, Lutzville, Vlêrmuisklip, near Vredendal, $31.39^{\circ} \mathrm{S}, 18.32^{\circ}$ E, 2 Oct. 1986, M.W. Mansell and J. Hoffman leg. (PPRI 86/650);

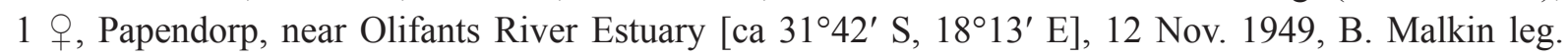

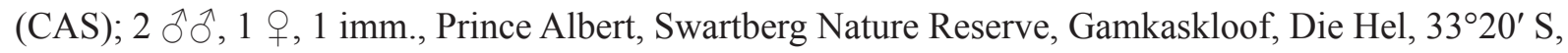
$21^{\circ} 40^{\prime}$ E, Sep. 1998, Z. van der Walt leg. (NMBA 8695, 8860); 1 ㅇ, 3 imm., Vanrhynsdorp, Van Rhyn's Pass, $31.6^{\circ} \mathrm{S}, 18.75^{\circ}$ E, 3 Oct. 1986, M.W. Mansell and J. Hoffman leg. (PPRI 86/648).

ZIMBABWE: 1 ㅇ, Pande Mine, 2230Ad, 9 Feb. 1994, F. Nyathi leg. (NMZB A/11518); 1 + 1 imm., Sentinel Ranch, Hunters Camp, 2229Ab, 16 Apr. 1990, Falcon Collage-NHMZ leg. (NMZB A/9940).

\section{Natural history}

Hexophthalma hahni have been collected in the Fynbos, Grassland, Karoo, Desert and Savanna biomes, by pitfall traps and by hand, in caves and from under rocks and logs.

\section{Distribution}

Hexophthalma hahni is distributed in Namibia, South Africa and southern Zimbabwe (Fig. 26).

Hexophthalma spatulata (Pocock, 1900)

Figs 16, 22-23, 25

Sicarius spatulatus Pocock, 1900: 321.

Sicarius spatulatus - Newlands 1986: 56. — Lotz 2012: 10.

Hexophthalma spatulata - Magalhaes et al. 2017: 853, figs 6A-D, 12C, 14D, 18H, 19D, 20E-F, 22A-E, 23D-F, 31H.

\section{Diagnosis}

Leg femora in both sexes with scoop-shaped enlarged dorsal setae (Fig. 23), differing from the setae of all other species. Female inner spermathecae consist of a broad chamber, extending anteriorly in numerous tubes, each ending in one or more spherical vesicles (Fig. 16), differing from all other species ,where the inner spermathecae are finger-like sacs. Male embolus ending in a broad, blunt triangular apex (Fig. 22), whereas the embolus of all the other species have thin apices.

\section{Material examined}

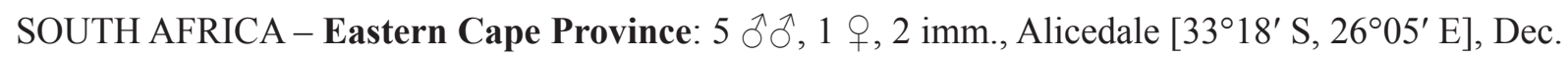
1914, F. Cruden leg. (AMSA); 1 , , same data as for preceding (AMSA 2525). - Western Cape Province: $1 \mathrm{O}^{\top}$, Borrelfontein, $8 \mathrm{~km} \mathrm{~W}$ of Gouritz River mouth, $34^{\circ} 20^{\prime} \mathrm{S}, 21^{\circ} 51^{\prime} \mathrm{E}, 4$ Mar. 2007, H. Leibel leg. (PPRI 2008/745); 1 đ, same data as for preceding but Nov. 2007-May 2008 (PPRI 2009/5601); 1 ठ,

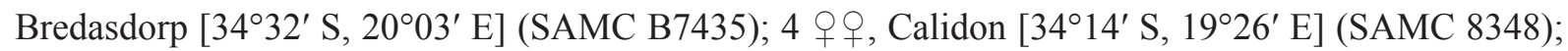

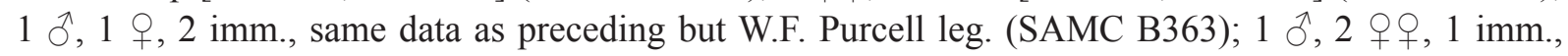

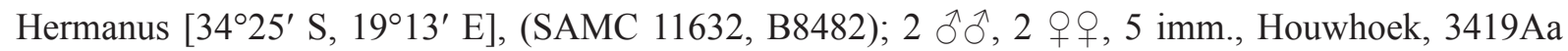

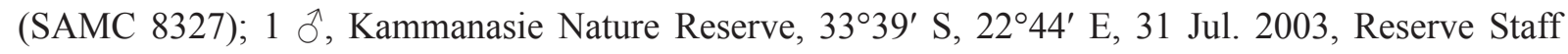
leg. (PPRI 2010/2403); 1 ㅇ, Piquetberg, 3218Dd (SAMC B8490); 1 q, Swellendam, Tradows Pass $\left[33^{\circ} 58^{\prime} \mathrm{S}, 20^{\circ} 42^{\prime} \mathrm{E}\right]$ (SAMC B6767). 


\section{Natural history}

Hexophthalma spatulata have been collected in a Fynbos biome.

\section{Distribution}

Hexophthalma spatulata is distributed along the southern coastal areas of South Africa (Fig. 25).

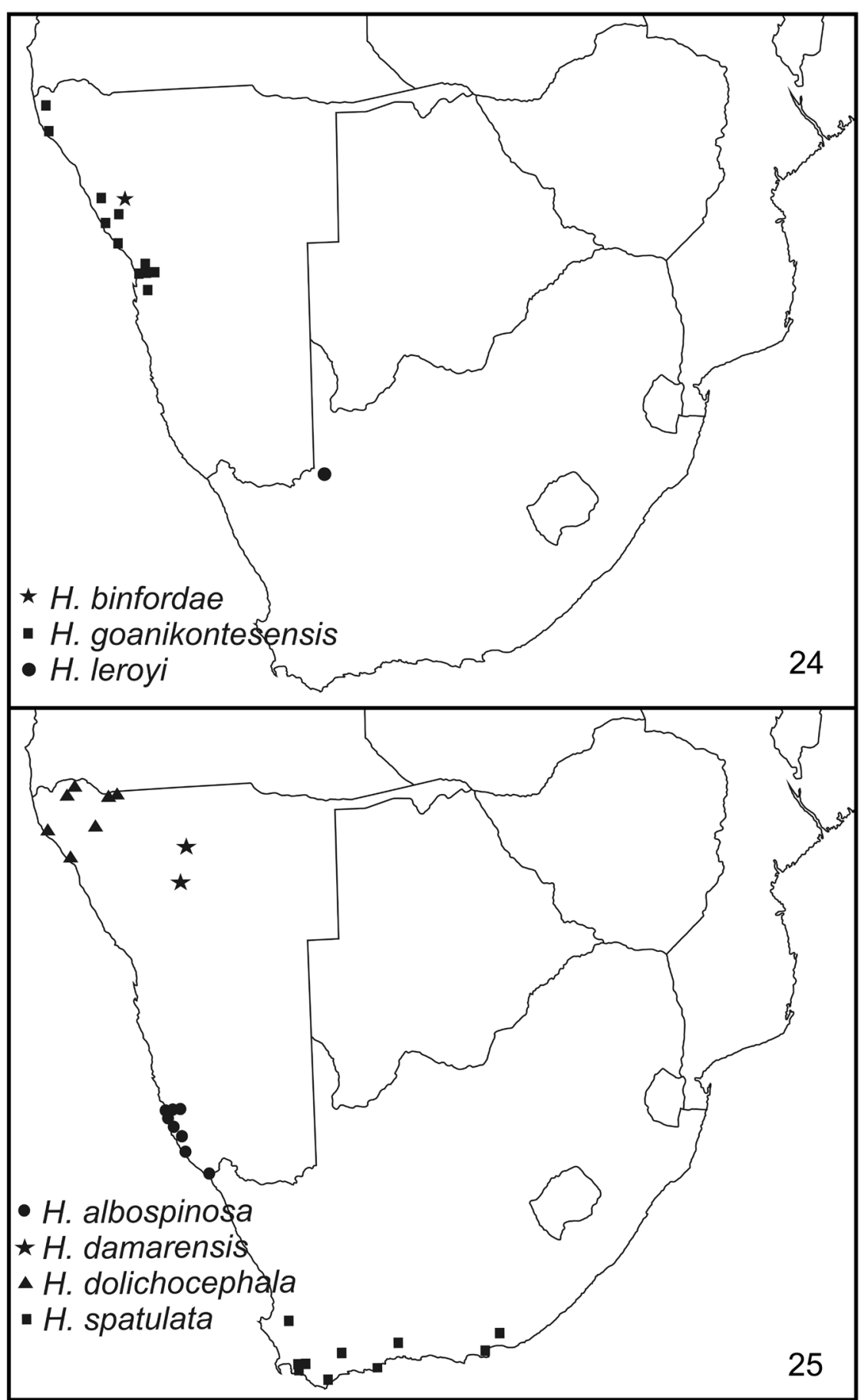

Figs 24-25. Distribution maps for species of Hexophthalma Karsch, 1879. 24. H. goanikontesensis sp. nov., H. binfordae sp. nov. and H. leroyi sp. nov. 25. H. albospinosa (Purcell, 1908, H. damarensis (Lawrence, 1928), H. dolichocephala (Lawrence, 1928) and H. spatulata (Pocock, 1900). 


\section{Discussion}

Species of Hexophthalma, the former African Sicarius, have raised interest because of their supposed medical importance (Newlands \& Atkinson 1988; Van Aswegen et al. 1997). The venom of some of the species has even been considered as one of the strongest spider venoms (Newlands 1986), but this has never been corroborated. Studies on this topic, mainly on the interspecific variation of the venom, which can be very large (Müller et al. 2012), are particularly difficult, because the identification of the species is virtually impossible. The study of Zobel-Thropp et al. (2010) is symptomatic: it was hindered by the lack of systematic knowledge and had to use approximate identifications like Sicarius cf. hahni and $S$. cf. damarensis. The present study has made a step forward, but it is clear that more efforts will be needed to facilitate studies on this genus, be it systematic, ecological or medical. In several species the males are still unknown, more species are to be expected and the monophyly of the genus is questionable because of the morphologically aberrant $H$. spatulata. Future studies will therefore have to be carried out along several axes. In the first place, further collecting will be needed to fill the gaps outlined above. Molecular studies in combination with detailed morphological assessments will be necessary to outline the interspecific relationships and the phylogenetic placement of the group, mainly in connection with its possible polyphyly.

\section{Acknowledgments}

I am very grateful to the institutions mentioned in the text for the loan of their specimens. The Council and Director of the National Museum Bloemfontein are also acknowledged for supporting this project.

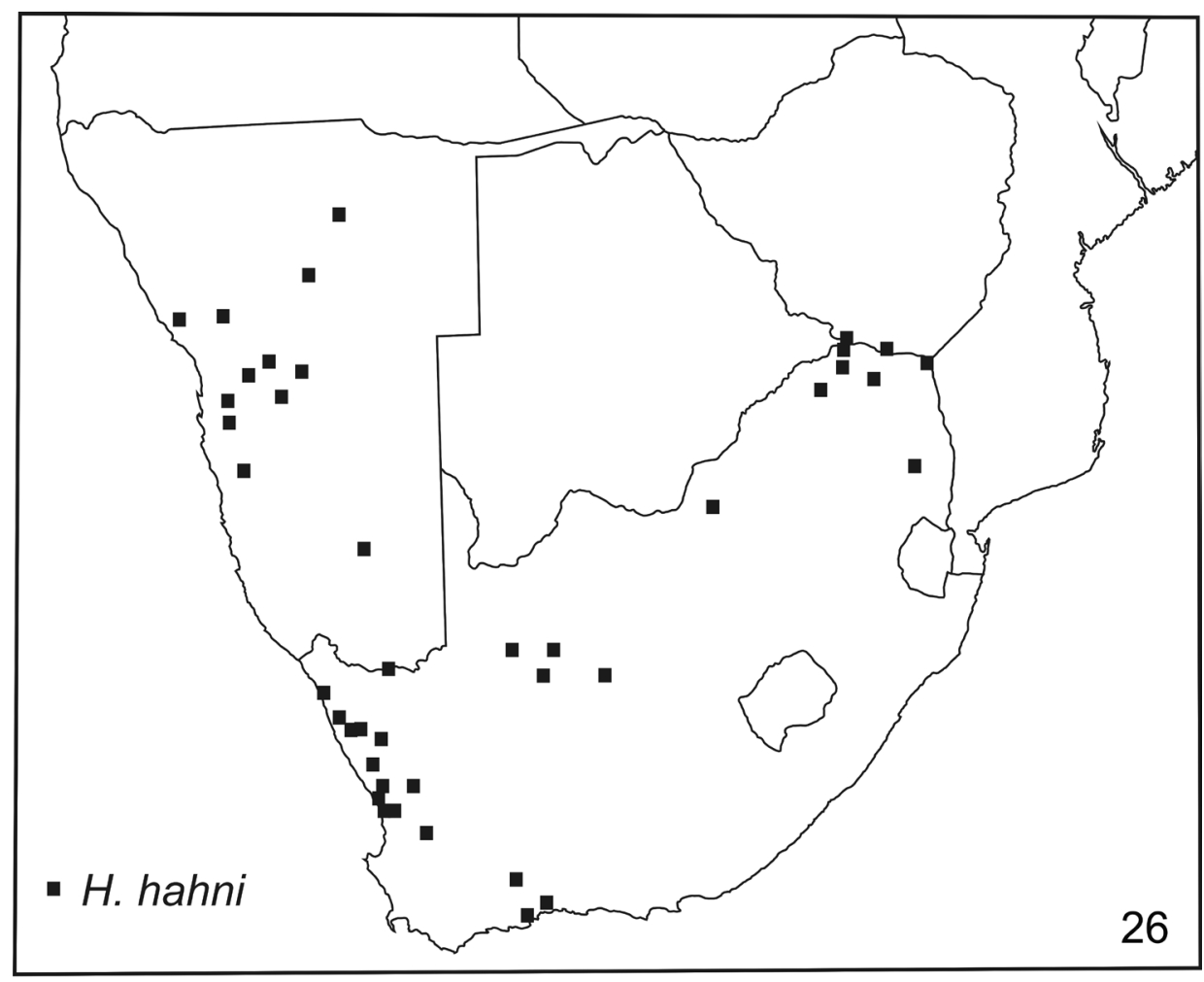

Fig. 26. Distribution map for Hexophthalma hahni (Karsch, 1878). 
LOTZ L.N., Spider genus Hexophthalma in the Afrotropical region

\section{References}

Karsch F. 1878. Exotisch-araneologisches. Zeitschrift für die gesammten Naturwissenschaften 51: $323-$ 333.

Karsch F. 1879. Sieben neue Arachniden von St Martha. Entomologische Zeitung herausgegeben von dem Entomologische Vereine zu Stettin 40: 106-109.

Available from https://biodiversitylibrary.org/page/9188954 [accessed 23 Mar. 2018].

Lawrence R.F. 1928. Contributions to a knowledge of the fauna of South-West Africa VII. Arachnida (Part 2). Annals of the South African Museum 25: 217-312.

Available from https://biodiversitylibrary.org/page/40656433 [accessed 23 Mar. 2018].

Lawrence R.F. 1938. Transvaal Museum Expedition to South-West Africa and Little Namaqualand, May to August 1937. Spiders. Annals of the Transvaal Museum 19: 215-226.

Lotz L.N. 2012. Present status of Sicariidae (Arachnida: Araneae) in the Afrotropical region. Zootaxa 3522: $1-41$.

Magalhaes I.L.F., Brescovit A.D. \& Santos A.J. 2017. Phylogeny of Sicariidae spiders (Araneae: Haplogynae), with a monograph on Neotropical Sicarius. Zoological Journal of the Linnean Society 179 (4): 767-864.

Müller G.J., Wium C.A., Marks C.J., Du Plessis C.E. \& Veale D.J.H. 2012. Spider bite in South Africa: diagnosis and management. Continuing Medical Education 30: 382-392. Available from http://www.cmej.org.za/index.php/cmej/article/view/2547/2582 [accessed 23 Mar. 2018].

Newlands G. 1986. Necrotic Arachnidism in Southern Africa. Unpublished Ph.D. thesis. University of the Witwatersrand, Johannesburg.

Newlands G. \& Atkinson P. 1988. Review of southern African spiders of medical importance, with notes on the signs and symptoms of envenomation. South African Medical Journal 73: 235-239.

Pocock R.I. 1900. Some new Arachnida from Cape Colony. Annals and Magazine of Natural History, Series 76 (31): 316-333. https://doi.org/10.1080/00222930008678382

Purcell W.F. 1908. Araneae (1). In: Schultze L. Forschungsreise im westlichen und zentralen Südafrika ausgeführt in den Jahren 1903-1905. Denkschrift der medicinisch-naturwissenschaftlichen Gesellschaft, Jena 13: 203-246. Available from https://biodiversitylibrary.org/page/37102545 [accessed 23 Mar. 2018].

Simon E. 1893. Famille Filistatidae. Histoire naturelle des Araignées 1: 255-488. https://biodiversitylibrary.org/page/38670557

Van Aswegen G., Van Rooyen J.M., Van der Nest D.G., Veldman F.J., De Villiers T.H. \& Oberholzer G. 1997. Venom of a six-eyed crab spider, Sicariustestaceus (Purcell, 1908), causes necrotic and haemorrhagic lesions in the rabbit. Toxicon 35: 1149-1152. https://doi.org/10.1016/S0041-0101(96)00203-6

World Spider Catalog 2017. World Spider Catalog. Version 18.0. Natural History Museum Bern. Available from https://wsc.nmbe.ch/ [accessed on 3 April 2017].

Zobel-Thropp P.A., Bodner M.R. \& Binford G.J. 2010. Comparative analyses of venoms from American and African Sicarius spiders that differ in sphingomyelinase D activity. Toxicon 55: 1274-1282. https://doi.org/10.1016/j.toxicon.2010.01.019

Manuscript received: 18 April 2017

Manuscript accepted: 11 September 2017 
Published on: 10 April 2018

Topic editor: Rudy Jocqué

Desk editor: Chloë Chester

Printed versions of all papers are also deposited in the libraries of the institutes that are members of the EJT consortium: Muséum national d'Histoire naturelle, Paris, France; Botanic Garden Meise, Belgium; Royal Museum for Central Africa, Tervuren, Belgium; Natural History Museum, London, United Kingdom; Royal Belgian Institute of Natural Sciences, Brussels, Belgium; Natural History Museum of Denmark, Copenhagen, Denmark; Naturalis Biodiversity Center, Leiden, the Netherlands; Museo Nacional de Ciencias Naturales-CSIC, Madrid, Spain; Real Jardín Botánico de Madrid CSIC, Spain. 